\title{
Lympho-Epithelial Carcinoma of the Orbit
}

\section{Asmae Touil, Sarah Elabbassi, Kebdani Tayeb and Noureddine Benjaafar}

Department of Radiation Oncology, National Institute of Oncology, Mohamed 5 University in Rabat, Morocco

Corresponding author: Touil Asmae, Resident of Radiation Oncology, Department of Radiotherapy, National Institute of Oncology, Mohamed 5 University in Rabat, Morocco, Tel: +212661696335; E-mail: asmaetouil@gmail.com

Received: 13 November 2016; Accepted: 17 November 2016; Published: 21 November 2016

Citation: Touil A, Elabbassi S, Tayeb K, et al. Lympho-Epithelial Carcinoma of the Orbit. Arch Can Res. 2016, 4: 4.

\section{Case Blog}

In our hospital, a one patient had 75 years old male and from Morocco was referred for us for realized the exophthalmia, conjunctival hyperhemia and visual disturbance. The biopsy was revealed a lympho-epithelial carcinoma; the patient has received a chemoradiation therapy. the clinical response was complete.

The same patient male was referred to our hospital for exophtalmia, conjunctival hyperhemia and visual disturbance. Facial magnetic resonance imaging reveled an extra-conical mass in the left orbit with extension to cavernous sinus and infra temporal fossa, the processus encompasses optic nerve and ocular muscles.

A nasofibroscopy with assisted video was realize and eliminate all lesions of cavum and nasal cavity, after that the diagnosis of orbital lymphoepithelial carcinoma was confirmed by immunohistochemical studies, after trans ethmoïdal biopsy.

The workup, including bone scan, abdominal ultrasonography and thoracic radiography, found no evidence of metastatic disease.

Surgery was not planned because of the local extension of the tumor. Our patient was managed by chemoradiation therapy. The patient had 6 cycles weekly of carboplatin AUC2 and $70 \mathrm{~Gy}$ in 35 fractions, $2 \mathrm{~Gy} /$ fraction.

Finally, a quasi complete regression of the tumor was considered as a good clinical response (Figures $\mathbf{1}$ and $\mathbf{2}$ ).

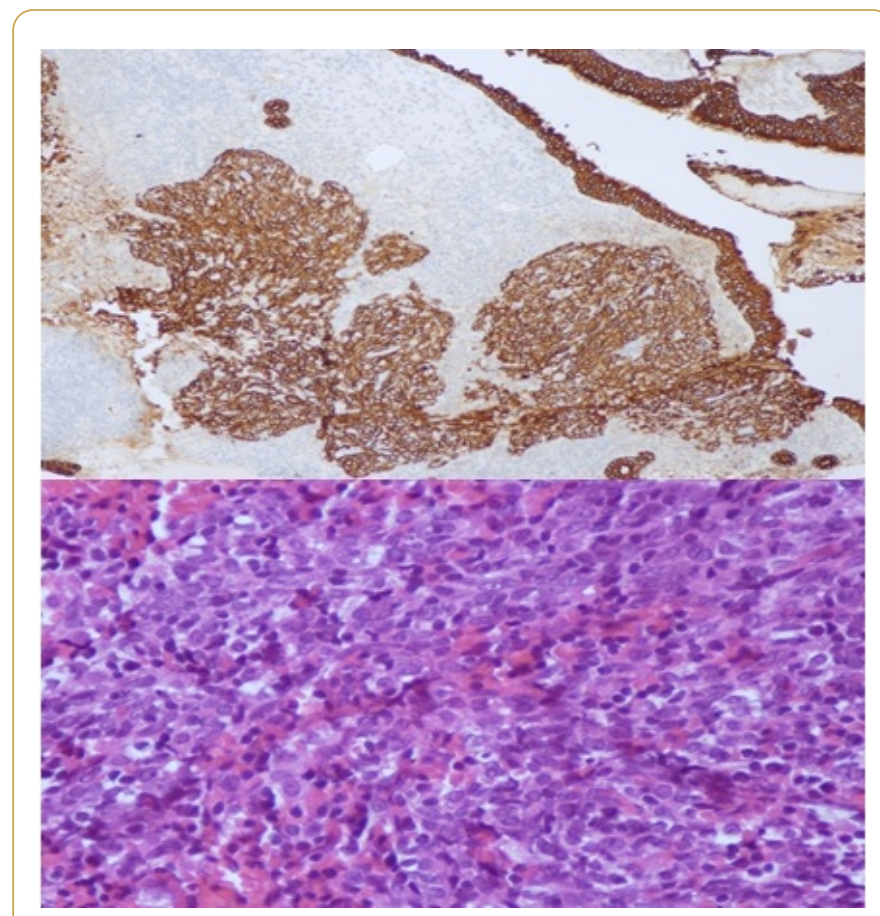

Figure 1 Syncytial wide proliferation of large cells with vesicular and nucleus nucleolus, in lymphoid stroma (HEx10).

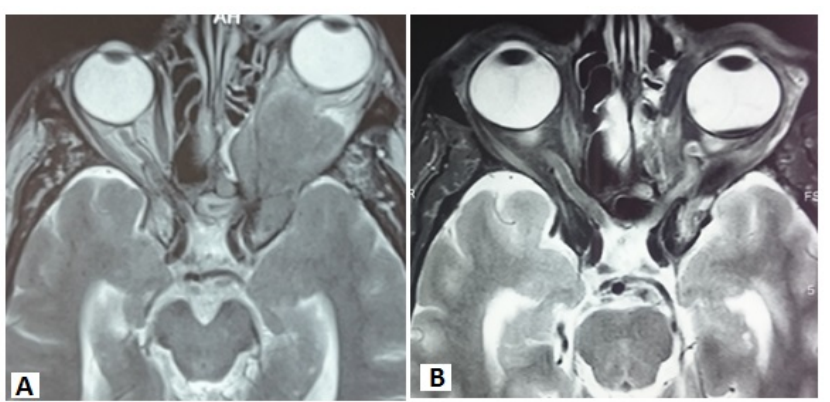

Figure 2 a: Facial MRI showing a extra-conical mass encompassing optic nerve and ocular muscles; b: evaluation one year after chemoradiation. 